\title{
Uso do tanino como fotossensibilizador na terapia fotodinâmica contra Enterococcus
}

\author{
faecalis \\ Use of tannin as a photosensitizer in photodynamic therapy against Enterococcus faecalis \\ Uso de tanino como fotosensibilizador en terapia fotodinámica contra Enterococcus faecalis
}

Recebido: 14/08/2021 | Revisado: 28/08/2021 | Aceito: 01/09/2021 | Publicado: 04/09/2021

Paula Vanessa Da Silva

ORCID: https://orcid.org/0000-0002-9251-9912 Faculdades Integradas de Patos, Brasil E-mail: paula_vanessa_ahotmail.com

Maria Cleide Azevedo Braz

ORCID: https://orcid.org/0000-0002-0290-6987 Centro Universitário de Patos, Brasil E-mail: cleide.braz2011@gmail.com

André Hayato Saguchi

ORCID: https://orcid.org/0000-0002-1903-4986 Universidade Cruzeiro do Sul, Brasil E-mail: andre_saguchi@yahoo.com.br

Diego Portes Vieira Leite ORCID: https://orcid.org/0000-0003-4936-3649 Universidade Cruzeiro do Sul, Brasil

E-mail: odontoportescursos@gmail.com

Ângela Toshie Araki Yamamoto

ORCID: https://orcid.org/0000-0003-4402-7531 Universidade Cruzeiro do Sul, Brasil

E-mail: angela.araki@cruzeirodosul.edu.br

\begin{abstract}
Resumo
O objetivo deste estudo foi avaliar a redução microbiana após a terapia fotodinâmica como coadjuvante ao preparo químico-cirúrgico (PQC) utilizando o tanino hidrolisável como fotossensibilizador, em canais radiculares de 60 dentes bovinos contaminados com Enterococcus faecalis. Dividiu-se os dentes em seis grupos: grupo NAOCl, onde utilizouse apenas o $\mathrm{NaOCl}$ a 2,5\%; grupo PDT/AM, utilizou-se a PDT com azul de metileno como fotossensibilizador; grupo Tan, apenas tanino foi utilizado; grupo PDT/Tan, utilizou-se PDT com tanino como fotossensibilizador; grupo $\mathrm{NaOCl} / \mathrm{PDT} / \mathrm{Tan}, \mathrm{NaOCl} 2,5 \%$ foi associado a PDT com tanino; e no grupo NaOCl/PDT/AM, onde associou-se ao $\mathrm{NaOCl} 2,5 \%$ o PDT com azul de metileno. Para a PDT usou-se $3 \mathrm{ml}$ do fotossensibilizador na concentração de $0,005 \%$ por $5 \mathrm{~min}$ (tempo de pré-irradiação). Para a irradiação foi utilizado um laser semicondutor portátil (Laser DUO ${ }^{\circledR}$, GaAlAs, InGaAlP), comprimento de onda de $660 \mathrm{~nm}$, potência de $100 \mathrm{~mW}$, totalizando 1,8 J de energia e tempo de irradiação de 180s. Foram realizadas três coletas microbiológicas, denominadas inicial (antes do PQC), intermediária (após o PQC) e final (7 dias após o PQC). Após as coletas, fez-se o plaqueamento para a contagem das UFC's. Os resultados foram expressos através das medidas estatísticas: média, desvio padrão, mediana, quartis e valores mínimo e máximo. Foi utilizado o teste F (ANOVA), as comparações múltiplas de Tamanho e o teste de Kruswal-Wallis. A verificação da hipótese de normalidade foi realizada através do teste de Shapiro-Wilk. O nível de significância estabelecido foi de 0,05 . Concluiu-se que terapia fotodinâmica, utilizando o tanino hidrolisável como fotossensibilizador mostrou-se eficaz como coadjuvante no preparo químico-cirúrgico na redução microbiana.
\end{abstract}

Palavras-chave: Terapia fotodinâmica; Endodontia; Corantes; Tanino.

\begin{abstract}
The present study aimed to evaluate the microbial reduction after photodynamic therapy as an adjunct to chemicalsurgical preparation (CSP), using the hydrolysable tannin as a photosensitizer in root canals of bovine teeth infected with Enterococcus faecalis. 60 teeth, divided into six groups: group $\mathrm{NaOCl}$, only $\mathrm{NaOCl} 2.5 \%$ was used; group PDT/MB, PDT with methylene blue as a photosensitizer was used; group Tan, only tannin was used as photosensitizer; group PDT/Tan, where only tannin PDT was used as photosensitizer; group NaOCl/PDT/Tan, $\mathrm{NaOCl} 2.5 \%$ was associated to tannin PDT; and group $\mathrm{NaOCl} / \mathrm{PDT} / \mathrm{MB}, \mathrm{NaOCl} 2.5 \%$ was associated to PDT with methylene blue. For PDT, $3 \mathrm{ml}$ of the photosensitizer was used at a $0,005 \%$ concentration for $5 \mathrm{~min}$ (pre- irradiation time). For the irradiation, a portable semiconductor laser (Laser DUO®, GaAlAs, InGaAlP) was used, wavelength of $660 \mathrm{~nm}$, power of $100 \mathrm{~mW}$, totaling $1.8 \mathrm{~J}$ of energy and $180 \mathrm{seg}$ of irradiation time. Three microbiological collections were performed, called initial (before SCP), intermediate (after CSP) and final (7 days after CSP). After the collections, plating was done for counting
\end{abstract}


the CFU's. The results were expressed as statistical measures: mean, standard deviation, median, quartiles and minimum and maximum values. The F-test (ANOVA) for multiple comparisons and the Tamanhe of Kruswal-Wallis test were used. The verification of the hypothesis of normality was performed using the Shapiro-Wilk test. The significance level was set at 0,05 . It was concluded that photodynamic therapy, using hydrolysable tannin as a photosensitizer, proved to be effective as an adjunct to chemical-surgical preparation in microbial reduction.

Keywords: Photodynamic therapy; Endodontics; Dyes; Tannin.

\section{Resumen}

El objetivo de este estudio fue evaluar la reducción microbiana después de la terapia fotodinâmica (TFD) como complemento de la preparación químico-quirúrgica (PQQ) utilizando tanino hidrolizable como fotosensibilizador en conductos radiculares de 60 dientes bovinos contaminados con Enterococcus faecalis. Los dientes se dividieron en seis grupos: grupo $\mathrm{NaOCl}$, donde solo se utilizó $\mathrm{NaOCl}$ al 2,5\%; grupo TFD/AM, se utilizó TFD con azul de metileno como fotosensibilizador; grupo Tan, solo se utilizó tanino; grupo TFD/Tan, PDT con tanino se utilizó como fotosensibilizador; grupo $\mathrm{NaOCl} / \mathrm{PDT} / \mathrm{Tan}, 2,5 \%$ de $\mathrm{NaOCl}$ se asoció con TFD con tanino; y en el grupo NaOCl/TFD/AM, donde el $\mathrm{NaOCl}$ al 2,5\% se asoció con TFD con azul de metileno. Para la TFD se utilizaron $3 \mathrm{ml}$ de fotosensibilizador a una concentración de $0,005 \%$ durante $5 \mathrm{~min}$ (tiempo de pré-irradiación). Para la irradiación se utilizó un láser semiconductor portátil (Laser DUO®, GaAlAs, InGaAlP)- 660nm, 100 mW, 1.8 J y tiempo de irradiación de 180s. Se realizaron tres recolecciones microbiológicas, denominadas inicial (anterior a PQQ), intermedia (después de PQQ) y final (7 días después de PQQ). Después, se realizó el plateado para contar las UFC. Los resultados se expresaron mediante medidas estadísticas: media, desviación estándar, mediana, cuartiles y valores mínimo y máximo. Se utilizó la prueba $\mathrm{F}$ (ANOVA), comparaciones múltiples de tamaño y la prueba de Kruswal-Wallis. La verificación de la hipótesis de normalidad se realizó mediante la prueba de Shapiro-Wilk. El nivel de significancia establecido fue de 0,05. Se concluyó que la terapia fotodinámica, utilizando tanino hidrolizable como fotosensibilizador, resultó ser eficaz como coadyuvante en la preparación químico-quirúrgica para la reducción microbiana.

Palabras clave: Terapia fotodinámica; Endodoncia; Tintes; Tanino.

\section{Introdução}

Erradicar ou reduzir a população microbiana no interior do canal radicular é o objetivo do tratamento endodôntico, permitindo a prevenção ou a cura da periodontite apical (Orstavik, 1996).

O Enterococcus faecalis (E. faecalis), microrganismo anaeróbio facultativo, tem sido a espécie mais prevalente em casos de insucesso endodôntico. As técnicas atuais para a desinfecção do Sistema de Canais Radiculares (SCR), incluem a instrumentação, a irrigação com agentes antimicrobianos e a medicação intracanal. No entanto, novos procedimentos para aprimorar a desinfecção do SCR vêm sendo desenvolvidos, dentre os quais o uso do laser de baixa potência associado ao fotossensibilizador (FS) na terapia fotodinâmica (Bonsor \& Pearson, 2006; Fonseca et al., 2008; Garcez et al., 2008; Seal et al., 2002; Siqueira \& Rocas, 2005).

A Terapia Fotodinâmica, também conhecida como PDT, acrônimo de Photodynamic Therapy, surge como uma promissora terapia antimicrobiana. Envolve a utilização de um fotossensibilizador (corante), que é ativado pela luz de um específico comprimento de onda na presença de oxigênio. Embora que, na endodontia, a solução de azul de metileno tem sido o principal FS utilizado como alvo para microrganismos da microbiota endodôntica (Konopka \& Goslinski, 2007), os fotossensibilizadores derivados das fenotiazinas têm sido amplamente empregados nas pesquisas envolvendo PDT

Taninos, do francês tanin, são os compostos fenólicos, corantes naturais que estão entre os maiores constituintes ativos derivados dos vegetais, justificando o uso tradicional das plantas como antiinflamatórios e cicatrizantes. Quando processados, originam um precipitado vermelho e, em solução, desenvolvem coloração verde ou azul (Asongalem et al., 2004).

Com o propósito de buscar novas formas terapêuticas, que apresentem menor toxicidade, maior potencial antimicrobiano, sobretudo contra o E. faecalis, e baixo custo no tratamento endodôntico, o objetivo deste trabalho foi quantificar a redução da carga bacteriana de canais radiculares de dentes bovinos contaminados por uma cepa (isolado endodôntico) de $E$. faecalis, aplicando a PDT, como coadjuvante ao preparo químico-cirúrgico (PQC), utilizando o tanino como FS. 


\section{Metodologia}

O experimento foi aprovado pelo Comitê de Ética em Pesquisa com Animais da Universidade Cruzeiro do Sul com protocolo 041/2014 e realizado no laboratório do curso de Biomedicina do Centro Universitário (UNIFIP) Patos/PB.

\section{Preparo dos espécimes}

Foram selecionados 60 incisivos bovinos hígidos com ápices completamente formados. Os espécimes foram limpos com raspadores periodontais para remoção de tecido periodontal ou ósseo aderidos à superfície radicular e com profilaxia com taça de borracha, pasta profilática e pedra-pomes. As coroas dos dentes foram removidas na junção amelocementária com disco diamantado mantendo os remanescentes radiculares com $21 \mathrm{~mm}$ de comprimento. Após esvaziamento do conteúdo pulpar com limas tipo K90 com soro fisiológico e realização da odontometria, os ápices foram vedados com resina epóxi Araldite (Brascola, São Paulo, SP) de endurecimento rápido a fim de prevenir o extravasamento da cultura bacteriana após a sua contaminação (Dametto et al., 2005). Os espécimes foram colocados em frascos contendo Brain Heart Infusion (BHI, Oxoid; Basinkstoke, UK) estéril e autoclavados a $120^{\circ} \mathrm{C}, 1 \mathrm{~atm}$, por $20 \mathrm{~min}$. Permaneceram incubados a $37^{\circ} \mathrm{C}$ por $24 \mathrm{~h}$ para confirmação da esterilidade.

Culturas puras de Enterococcus faecalis (ATCC 29212) em caldo de BHI foram utilizadas como contaminantes dos espécimes. Colônias isoladas foram suspensas em tubos com tampas rosqueáveis contendo $5 \mathrm{ml}$ de BHI. Esta suspensão foi agitada mecanicamente e ajustada em espectrofotômetro com absorbância de $800 \mathrm{~nm}$, até obter-se a concentração equivalente a 1,0 da escala McFarland (3,0 x $10^{8}$ bactérias $\left./ \mathrm{ml}\right)$. Os frascos contendo os espécimes estéreis foram abertos em uma câmara de fluxo laminar. Pipetas estéreis removeram 5,0 ml de BHI estéril e introduziram 5,0ml da suspensão de $E$ faecalis. Os frascos foram vedados e incubados em estufa a $37^{0} \mathrm{e}$ atmosfera de $10 \%$ de $\mathrm{CO}_{2}$ por 7 dias, com trocas de 1,0 $\mathrm{ml}$ de $\mathrm{BHI}$ contaminado por 1,0 $\mathrm{ml}$ de BHI estéril, a cada 2 dias, a fim de se evitar a saturação do meio. O crescimento bacteriano durante o período de incubação foi constatado pela presença de turbidez do meio (Dametto et al., 2005; Gomes et al., 2001; Menezes et al., 2004).

Todo experimento foi realizado em câmara de fluxo laminar para evitar contaminação cruzada. Durante a realização do $\mathrm{PQC}$, todos os espécimes de cada grupo foram retirados dos frascos por meio de uma pinça estéril e dispostos em uma plataforma metálica estéril, com pinos laterais rosqueáveis (Figura 1).

Figura 1 - Dentes bovinos dispostos na plataforma metálica.

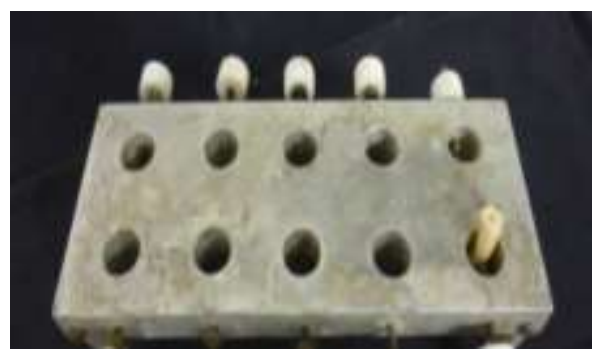

Fonte: Autores.

\section{Grupos experimentais}

Os 60 espécimes foram distribuídos em 6 grupos experimentais $(\mathrm{n}=10)$ de acordo com o protocolo de desinfecção durante o PQC (Tabela 1).

Para a instrumentação dos espécimes de todos os grupos foi utilizada a técnica manual escalonada coroa-ápice com limas tipo K file (Dentsply Maillefer; Baillaigues, Switzerland) até a lima \#120 com comprimento real de trabalho de $20 \mathrm{~mm}$, de acordo com trabalho prévio (Dametto et al., 2005). No entanto, os grupos se diferiram pela solução irrigadora, fotossensibilizador 
e uso de PDT ou não. Nos grupos em que houve realização de PDT, foram utilizados $3 \mathrm{ml}$ do fotossensibilizador na concentração de $0,005 \%$ e período de pré-irradiação de 5 minutos. A irradiação na entrada dos canais se deu com um laser semicondutor portátil (Laser DUO ${ }^{\circledR}$, GaAlAs, InGaAlP, MM OPTICS LTDA, São Carlos, SP - Brasil), ajustado em 660nm, 100mW, 1,8J e 180 segundos de irradiação, seguida irrigação com 10ml de soro fisiológico para remoção do corante (Gomes et al., 2003). As soluções empregadas durante o PQC, fotossensibilizador e emprego de PDT ou não são descritas a seguir:

\section{a) Grupo NaOCl: NaOCL a 2,5\%}

Durante o PQC, os canais foram irrigados com $3 \mathrm{ml}$ do $\mathrm{NaOCl}$ a 2,5\% a cada troca de limas, seguidos de irrigação com $10 \mathrm{ml}$ de soro fisiológico.

\section{b) Grupo PDT/AM: PDT com Azul de Metileno}

Durante o PQC, os canais foram irrigados com $3 \mathrm{ml}$ de soro fisiológico a cada troca de lima e 10ml de soro fisiológico na irrigação final. Após PQC, os canais foram preenchidos com 3ml de azul de metileno a 0.005\% (Chimiolux, Hypofarma, Belo Horizonte, Brasil) seguindo-se da irradiação conforme protocolo anteriormente citado. $\mathrm{O}$ azul de metileno foi totalmente retirado do canal através de irrigação com $10 \mathrm{ml}$ de soro fisiológico.

c) Grupo Tan: Tanino

Durante o PQC, os canais foram irrigados com 3ml de soro fisiológico a cada troca de lima e 10ml de soro fisiológico na irrigação final. Após PQC, os canais foram preenchidos com 3ml do extrato hidrolizável de tanino a 0,005\% durante 5minutos. Após esse tempo, todo o tanino foi removido dos canais através de uma irrigação com $10 \mathrm{ml}$ de soro fisiológico.

\section{d) Grupo PDT/Tan: PDT com Tanino}

Durante o PQC, os canais foram irrigados com $3 \mathrm{ml}$ de soro fisiológico a cada troca de lima e 10ml de soro fisiológico na irrigação final. Após PQC, os canais foram preenchidos com 3ml do extrato de tanino a 0,005\% seguindo-se da irradiação conforme protocolo anteriormente citado. Posteriormente, todo o tanino foi removido dos canais através de uma irrigação com $10 \mathrm{ml}$ de soro fisiológico.

\section{e) Grupo NaOCl/PDT/Tan: $\mathrm{NaOCl}+\mathrm{PDT}$ com Tanino}

Durante o PQC, os canais foram irrigados com $3 \mathrm{ml}$ do $\mathrm{NaOCl}$ a 2,5\% a cada troca de lima e $10 \mathrm{ml}$ de soro fisiológico na irrigação final. Após o preparo, os canais foram preenchidos com $3 \mathrm{ml}$ do extrato de tanino a 0,005\% seguindo-se da irradiação conforme protocolo anteriormente citado.

\section{f) Grupo NaOCl/PDT/AM: NaOCl+ PDT com Azul de Metileno}

Durante o PQC, os canais foram irrigados com $3 \mathrm{ml}$ do $\mathrm{NaOCl}$ a 2,5\% a cada troca de lima e $10 \mathrm{ml}$ de soro fisiológico na irrigação final. Após o preparo, os canais foram preenchidos com 3ml de azul de metileno 0.005\% (Chimiolux, Hypofarma, Belo Horizonte, Brasil) e PDT conforme descrição anterior. O azul de metileno foi totalmente retirado do canal através de irrigação com $10 \mathrm{ml}$ de soro fisiológico. 
Tabela 1 - Grupos experimentais (Distribuição dos grupos).

\begin{tabular}{lll}
\hline GRUPOS & N & SUBSTÂNCIAS \\
\hline $\mathrm{NaOCl}$ & 10 & Hipoclorito de Sódio 2,5\% \\
\hline PDT/AM & 10 & PDT com Azul de Metileno \\
\hline Tan & 10 & Tanino \\
\hline PDT/Tan & 10 & PDT com Tanino \\
\hline NaOCl/PDT/Tan & 10 & NaOCl 2,5\% + PDT com Tanino \\
\hline NaOCl/PDT/AM & 10 & NaOCl 2,5\% +PDT com Azul de Metileno \\
\hline
\end{tabular}

Fonte: Autores.

\section{Coletas microbiológicas}

Foram realizadas três coletas microbiológicas: antes do PQC (coleta inicial), imediatamente após o PQC (coleta intermediária) e após 7 dias da realização do PQC (coleta final).

\section{a) Coleta Inicial}

A coleta se deu antes do PQC com pontas de papel absorvente estéreis de tamanho FM que permaneceram no canal por 1 minuto e posteriormente depositadas em tubos individuais eppendorf.

b) Coleta Intermediária

Após PQC, os canais foram secos com pontas de papel absorvente estéreis que foram posteriormente depositadas em eppendorfs individuais.

Todos os espécimes submetidos ao tratamento foram preenchidos com BHI Agar, selados com guta-percha bastão para evitar que microrganismos presentes na superfície externa dos canais adentrassem o seu interior, e colocados em eppendorfs individuais contendo $60 \mu \mathrm{l}$ de BHI Agar estéril para manutenção da hidratação. É importante ressaltar que o BHI Agar atingiu até o terço médio da raiz para não infiltrar no selamento com guta-percha (Figura 2). Posteriormente, foram novamente incubados a $37^{\circ} \mathrm{C}$ em atmosfera de $10 \% \mathrm{CO}_{2}$ por 7 dias.

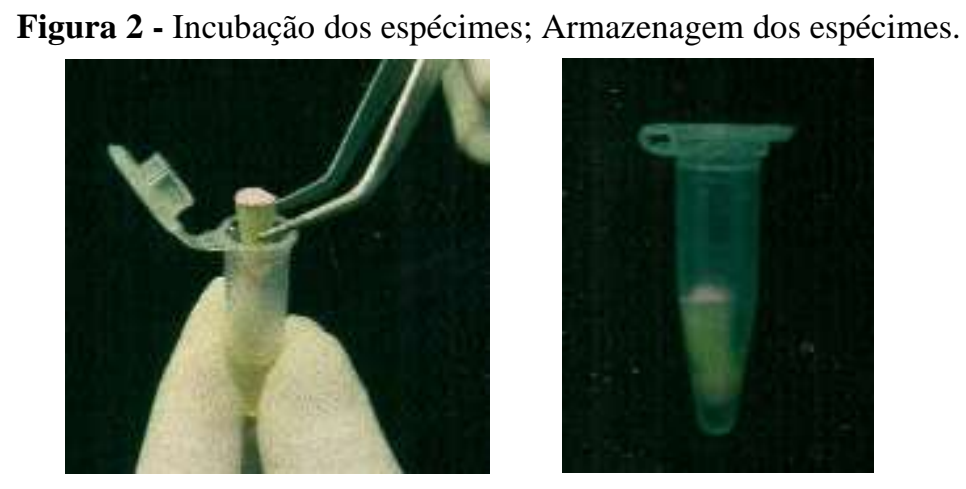

Fonte: Autores.

c) Coleta final

Após 7dias, o selamento cervical de guta percha foi removido e os canais radiculares foram novamente secos com pontas 
de papel absorvente estéreis e depositadas em eppendorfs individuais contendo 1ml de BHI estéril.

\section{Diluição e plaqueamento das coletas}

Após agitação mecânica, houve diluição das amostras coletadas na proporção de 1/10, 1/100, em BHI estéril. A seguir, $50 \mu 1$ da diluição $10^{-2}$ foram plaqueados em triplicata em BHI Agar e incubados a $37^{\circ} \mathrm{C}$ em atmosfera de $10 \%$ de $\mathrm{CO}_{2}$. Após o período de 48h, as colônias de microrganismos que estavam presentes nestas placas, uma vez constatada a pureza das colônias, através das características morfológicas típicas do grupo microbiano estudado, foram contadas, determinando-se as unidades formadoras de colônias (UFC). Para a obtenção das unidades formadoras de colônias por ml (UFC), foi necessária a multiplicação do número de UFC obtidas na contagem das placas em 2000 vezes, representando assim, o número de colônias descontando-se

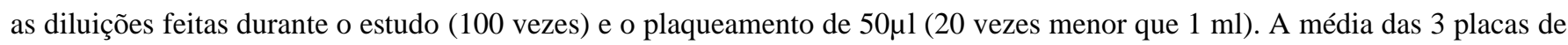
cada coleta foi utilizada como valor final da contagem (Figuras 3 e 4).

Figura 3 - Diluição e plaqueamento em triplicata.

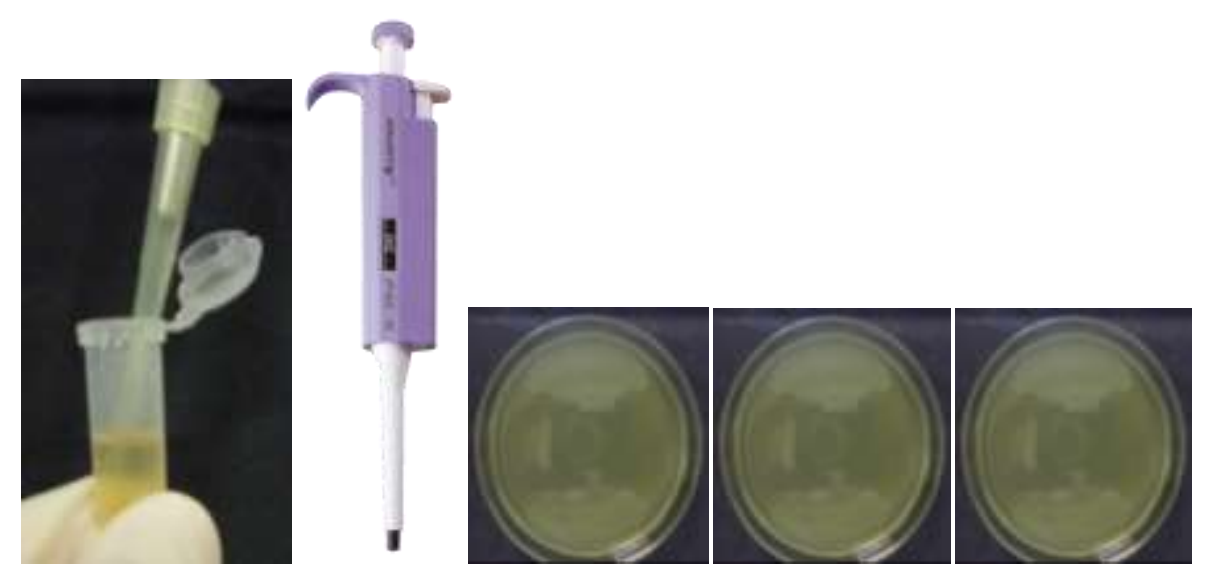

Fonte: Autores.

Figura 4 - Placas contendo UFC's.
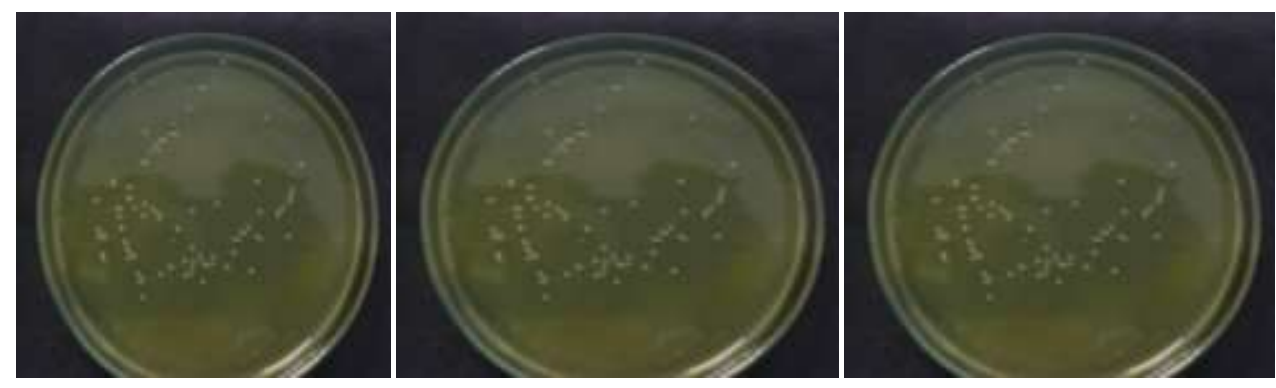

Fonte: Autores.

\section{Análise Estatística}

Os resultados foram expressos através das medidas estatísticas: média, desvio padrão, mediana, quartis (ou P25 e P75) e valores mínimo e máximo. Para a comparação entre os grupos em cada avaliação foi utilizado o teste F (ANOVA) em relação ao logaritmo decimal do número de colônias de bactérias e no caso de diferenças significativas foram utilizadas as comparações múltiplas de tamanho. Para a comparação entre os grupos em relação às variações percentuais entre a avaliação final com cada avaliação foi utilizado o teste de Kruskal-Wallis com comparações de múltiplas do teste, devido à falta de normalidade dos percentuais na maioria dos grupos. A verificação da hipótese de normalidade foi realizada através do teste de Shapiro-Wilk e 
para a verificação da hipótese de normalidade foi realizada através do teste $\mathrm{F}$ de Levene. A margem de erro utilizada nas decisões dos testes estatísticos foi de 5\%. Foi utilizado o programa SPSS (Statistical Package for the Social Sciences) na versão 21.

\section{Resultados}

Na Tabela 2 apresentam-se as estatísticas do número de bactérias (número de colônias x 2 x 10²) por avaliação e grupo. Desta tabela destaca-se: na avaliação inicial a média foi menos elevada no grupo PDT/AM $(56,93)$, seguido do grupo Tan $(81,20)$ e grupo $\mathrm{NaOCl}(84,53)$ e variou de 100,30 a 112,73 nos outros três grupos (PDT/Tan, NaOCl/PDT/Tan, NaOCl/PDT/AM); as três medianas menos elevadas ocorreram nos grupos: grupo $\mathrm{NaOCl}(37,83)$, grupo PDT/AM $(46,50)$ e grupo Tan $(50,00)$ e a mais elevada ocorreu no grupo PDT/Tan $(90,17)$, entretanto para a margem de erro fixada não se comprova diferença entre os grupos na avaliação inicial ( $\mathrm{p}>0,05)$.

$\mathrm{Na}$ avaliação intermediária a menor média foi registrada no grupo $\mathrm{NaOCl}(0,50)$, seguida do grupo $\mathrm{NaOCl} / \mathrm{PDT} / \mathrm{Tan}$ $(1,57)$ e a mais elevada ocorreu no grupo PDT/AM $(6,00)$, seguida do grupo Tan $(5,93)$; as medianas menos elevadas ocorreram nos grupos: $\mathrm{NaOCl}(0,33), \mathrm{NaOCl} / \mathrm{PDT} / \mathrm{Tan}(1,50)$ e $\mathrm{NaOCl} / \mathrm{PDT} / \mathrm{AM}(2,00)$ e as mais elevadas nos grupos Tan $(5,17)$ e PDT/AM $(4,17)$, diferenças estas que se mostraram significativas entre os grupos e através das comparações múltiplas (entre pares de grupos) se comprova diferença significativa entre cada um dos grupos $\mathrm{NaOCl}$ e $\mathrm{NaOCl} / \mathrm{PDT} / \mathrm{Tan}$ com cada um dos grupos PDT/AM e Tan.

$\mathrm{Na}$ avaliação final as médias e medianas foram correspondentemente menos elevadas nos grupos: grupo $\mathrm{NaOCl} / \mathrm{PDT} / \mathrm{Tan}$ (média de 0,67 em cada medida), grupo NaOCl/PDT/AM (média de 6,07 e mediana igual a 5,00) e grupo $\mathrm{NaOCl}$ (média de 11,93 e mediana de 5,33) e foram mais elevadas no grupo PDT/Tan (média de 51,80 e mediana de 47,43) e se comprova diferença significativa entre os grupos, sendo diferentes entre o grupo $\mathrm{NaOCl} / \mathrm{PDT} / \mathrm{Tan}$ com todos os grupos, do grupo $\mathrm{NaOCl} / \mathrm{PDT} / \mathrm{AM}$ com todos os grupos exceto com o grupo $\mathrm{NaOCl} / \mathrm{PDT} / \mathrm{AM}$, do grupo $\mathrm{NaOCl}$ com os grupos Tan e PDT/Tan e do grupo PDT/AM com o grupo PDT/Tan.

A variabilidade expressa através do desvio padrão de mostrou bastante elevada dede que para alguns grupos os valores do desvio padrão foram mais elevados dos que as médias correspondentes. 
Tabela 2 - Estatística do número de colônias (x 2 x 10²) segundo o grupo e o tempo de avaliação.

\begin{tabular}{|c|c|c|c|c|c|c|c|}
\hline Avaliação & Grupo & Média \pm DP & Mínimo & P25 & $\begin{array}{l}\text { Median } \\
\qquad a\end{array}$ & P75 & Máximo \\
\hline \multirow[t]{6}{*}{ 回 Inicial } & $\mathrm{NaOCl}$ & $84,53 \pm 113,91$ & 2,33 & 10,92 & 37,83 & 133,75 & 295,00 \\
\hline & PDT/AM & $56,93 \pm 26,34$ & 31,33 & 37,50 & 50,00 & 70,83 & 114,00 \\
\hline & Tan & $81,20 \pm 78,79$ & 33,00 & 45,25 & 46,50 & 97,25 & 295,00 \\
\hline & PDT/Tan & $111,00 \pm 80,55$ & 27,33 & 54,25 & 90,17 & 159,25 & 265,33 \\
\hline & $\mathrm{NaOCl} / \mathrm{PDT} / \mathrm{Tan}$ & $100,30 \pm 106,39$ & 12,67 & 29,00 & 65,00 & 147,00 & 295,00 \\
\hline & $\mathrm{NaOCl} / \mathrm{PDT} / \mathrm{AM}$ & $112,73 \pm 112,15$ & 20,67 & 26,25 & 68,00 & 238,25 & 295,00 \\
\hline Valor de p & & $p^{(1)}=0,353$ & & & & & \\
\hline \multirow[t]{6}{*}{$\begin{array}{l}\text { 圆 } \\
\text { Intermediária }\end{array}$} & $\mathrm{NaOCl}$ & $0,50 \pm 0,59$ (A) & 0,00 & 0,00 & 0,33 & 0,83 & 1,67 \\
\hline & PDT/AM & $6,00 \pm 4,09$ (B) & 3,00 & 3,67 & 4,17 & 7,25 & 16,67 \\
\hline & Tan & $5,93 \pm 2,10^{(\mathrm{B})}$ & 3,00 & 4,58 & 5,17 & 8,33 & 9,33 \\
\hline & PDT/Tan & $2,67 \pm 2,77^{(\mathrm{AB})}$ & 0,00 & 0,00 & 2,33 & 3,67 & 9,33 \\
\hline & $\mathrm{NaOCl} / \mathrm{PDT} / \mathrm{Tan}$ & $1,57 \pm 0,75^{(A)}$ & 0,33 & 1,00 & 1,50 & 2,08 & 3,00 \\
\hline & $\mathrm{NaOCl} / \mathrm{PDT} / \mathrm{AM}$ & $3,60 \pm 3,78^{(\mathrm{AB})}$ & 0,33 & 0,92 & 2,00 & 5,50 & 12,00 \\
\hline Valor de $p$ & & $p^{(1)}<0,001^{*}$ & & & & & \\
\hline \multirow[t]{6}{*}{ 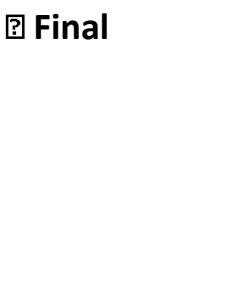 } & $\mathrm{NaOCl}$ & $11,93 \pm 16,86^{(\mathrm{AF})}$ & 1,00 & 1,75 & 5,33 & 14,75 & 55,33 \\
\hline & PDT/AM & $29,53 \pm 17,09(\mathrm{AC})$ & 11,33 & 19,58 & 27,17 & 30,83 & 74,33 \\
\hline & Tan & $33,07 \pm 18,35^{(B C D)}$ & 19,00 & 19,58 & 26,33 & 44,00 & 74,33 \\
\hline & PDT/Tan & $51,80 \pm 13,83^{(D)}$ & 38,00 & 40,58 & 47,33 & 63,58 & 80,33 \\
\hline & $\mathrm{NaOCl} / \mathrm{PDT} / \mathrm{Tan}$ & $0,67 \pm 0,52^{(E)}$ & 0,00 & 0,25 & 0,67 & 1,00 & 1,67 \\
\hline & $\mathrm{NaOCl} / \mathrm{PDT} / \mathrm{AM}$ & $6,07 \pm 2,96^{(F)}$ & 2,67 & 4,00 & 5,00 & 7,58 & 11,33 \\
\hline Valor de p & & $p^{(1)}<0,001^{*}$ & & & & & \\
\hline
\end{tabular}

(*): Diferença significativa ao nível de 5,0\%.

(1): Através do teste de F(ANOVA) com comparações de Tamanhe aplicados no logaritmo decimal no número de colônias de bactérias.

Obs.: Se todas as letras entre parênteses são distintas, comprova-se diferença significativa entre os grupos correspondentes.

Fonte: Autores.

A variabilidade entre a avaliação inicial e intermediária se mostrou reduzida se comparada com os valores das médias na variação inicial menos a intermediária oscilaram de baixa nos grupos NaOCl/PDT/Tan e NaOCl/PDT/AM a bastante elevados (valores do desvio padrão superiores aos valores das médias correspondentes). 
Tabela 3 - Estatísticas das variações percentuais entre a avaliação inicial com cada avaliação em relação ao número de colônias segundo o grupo.

\begin{tabular}{|c|c|c|}
\hline Grupo & Inicial - intermediária & Inicial - intermediária \\
\hline $\mathrm{NaOCl}$ & $98,55 \pm 2,13(99,60)^{(\mathrm{A})}$ & $-1,70 \pm 176,47(82,61)^{(A)}$ \\
\hline PDT/AM & $88,08 \pm 7,88(89,57)^{(\mathrm{B})}$ & $35,36 \pm 62,87(49,28)^{(\mathrm{B})}$ \\
\hline Tan & $89,10 \pm 5,70(88,89)^{(\mathrm{B})}$ & $39,74 \pm 45,85(54,18)$ \\
\hline PDT/Tan & $94,16 \pm 9,28(96,82)^{(\mathrm{A})}$ & $28,46 \pm 43,69(40,71)^{(\mathrm{B})}$ \\
\hline $\mathrm{NaOCl} / \mathrm{PDT} / \mathrm{Tan}$ & $96,51 \pm 2,95(97,26)^{(\mathrm{A})}$ & $98,33 \pm 1,78(98,93)^{(\mathrm{C})}$ \\
\hline $\mathrm{NaOCl} / \mathrm{PDT} / \mathrm{AM}$ & $94,48 \pm 6,04(97,64)^{(\mathrm{A})}$ & $88,76 \pm 8,61(88,19)$ \\
\hline Valor de $\mathrm{p}$ & $\mathrm{p}^{(1)}<0,001^{*}$ & $\mathrm{p}^{(1)}<0,001 *$ \\
\hline
\end{tabular}

(*): Diferença significativa ao nível de 5,0\%.

(1): Através do teste de Kruskal-Wallis com comparações de Tamanhe.

Obs.: Se todas as letras entre parênteses são distintas, comprova-se diferença significativa entre os grupos correspondentes. Fonte: Autores.

\section{Discussão}

Os dentes bovinos se assemelham estruturalmente aos dentes humanos, principalmente nas estruturas dos prismas de esmalte e do esmalte interprismático. Outros estudos utilizaram o dente bovino para examinar a penetração de bactérias nos túbulos dentinários e avaliar a ação antimicrobiana de algumas substâncias (Haapasalo et al., 2005).

O E. faecalis sobrevive em condições de escassez nutricional e em ausência de oxigênio, penetra nos túbulos dentinários e está associado ao insucesso do tratamento endodôntico (Evans et al., 2002; Gomes et al., 2001; Siqueira et al., 1997; Siren et al., 1997), sendo empregado em estudos que avaliaram a eficácia da PDT (Bergmans et al., 2008; Fonseca et al., 2008; Silva Garcez et al., 2006; Soukos et al., 2006). A cepa de E. faecalis empregada foi a isolada de canais radiculares, sendo, portanto, uma cepa selvagem (Zoletti et al., 2006). Entre os estudos que utilizaram o E. faecalis como marcador biológico, não existe consenso sobre o período ideal de incubação, podendo este variar de vinte e quatro horas a quatro semanas. Os presentes autores estabeleceram sete dias como período de incubação, como em estudos prévios (Dametto et al., 2005; Gomes et al., 2001; Menezes et al., 2004). Os valores obtidos com a coleta inicial comprovam que esse tempo foi suficiente para promover a colonização das amostras.

O protocolo para a realização da PDT ainda não está bem definido, existindo diversas variáveis, entre as quais o tipo e a concentração do Fotosenssibilizador (FS) a ser usado e os parâmetros do laser, bem como o tempo e a técnica de aplicação do FS e do laser. Os FS mais utilizados pelos autores que estudaram a TFD foram o azul de metileno (Fimple et al., 2008; Soukos et al., 2006), o azul de toluidina (Bergmans et al., 2008; Bonsor et al., 2006; Fonseca et al., 2008; Gomes et al., 2008; Seal et al., 2002; Williams et al., 2006) e os derivados do PEI-ce6 (Garcez et al., 2008; Garcez et al., 2007; Soukos et al., 1998).

Diversos corantes artificiais e naturais vêm sendo testados como FS na PDT, tanto para o tratamento do câncer, como para a redução de microrganismos. Corantes menos tóxicos, mais ressonantes com o comprimento de onda emitido pelos lasers têm sido o ideal de diversos pesquisadores. E quanto mais próximos deste ideal, estes agentes se mostrarem, mais a PDT realizará sua função na prática clínica endodôntica (Carson et al., 2005).

No presente estudo, foram testadas a eficácia de dois corantes: o azul de metileno, por ser o corante mais utilizado na PDT em Endodontia (Fimple et al., 2008; Wainwright, 2008) e o tanino hidrolizável por apresentar atividade promissora sem apresentar qualquer toxicidade e possuem propriedades fotofísicas favoráveis, com altos rendimentos do estado tripleto.

$\mathrm{Na}$ literatura, encontramos a utilização de diversas concentrações do azul de metileno, variando de $1 \mu \mathrm{g} / \mathrm{ml} \mathrm{a} 25 \mu \mathrm{g} / \mathrm{ml}$, 
bem como do azul de toluidina, variando de $12,5 \mu \mathrm{g} / \mathrm{ml}$ a $100 \mu \mathrm{g} / \mathrm{ml}$. Os dois FS empregados neste estudo, tanino e azul de metileno, foram utilizados na mesma concentração de $5 \mu \mathrm{g} / \mathrm{ml}$, mesma concentração em que o azul de metileno foi utilizado em outro estudo com o objetivo de padronizar o experimento (Gomes et al., 2008).

A fonte de radiação empregada foi o Twin laser, um laser diodo de baixa potência da MMOptics. Esse aparelho emite uma luz em dois intervalos espectrais: o visível (luz vermelha) e o infravermelho, com $660 \mathrm{~nm}$ e $780 \mathrm{~nm}$ de comprimento de onda respectivamente. Para esse experimento, foi aplicado o laser vermelho do Twin laser, que possui um meio ativo semicondutor de GaAlAs e potência máxima de $100 \mathrm{~mW}$.

Baseando-se no protocolo proposto em outro estudo que também utilizaram o Twin laser (Gomes et al., 2003), foi estabelecido em três minutos o tempo de pré-irradiação durante o qual o FS é deixado no canal, e em cinco minutos o tempo de aplicação da luz do laser na entrada do canal radicular, resultando numa dosagem total de 1,8 $\mathrm{J}$ de energia.

Ao testarem a eficácia da PDT com o azul de metileno como agente FS, Soukos et al. (2006) e Fimple et al. (2008) concluíram que essa terapia poderia ser um eficaz auxiliar no tratamento endodôntico convencional. Fimple et al. (2008), observaram uma redução da infecção de $80 \%$ em dentes contaminados por um biofilme multiespécies, enquanto Soukos et al. (2006) obtiveram uma redução de $97 \%$ em dentes contaminados por E. faecalis. Esta eficácia também foi observada no presente estudo, que mesmo o NaOCL a 2,5\% mostrando-se mais eficaz na redução microbiana entre as coletas inicial e intermediária, quando utilizou-se a PDT com azul de metileno como coadjuvante ao PQC houve uma redução de $96,51 \%$, entre as coletas inicial e final.

Resultados semelhantes foram observados nos estudos in vitro em que o azul de metileno foi aplicado como fotossensibilizador. Williams et al. (2006) observaram que PDT com azul de metileno foi eficaz em reduzir a contagem bacteriana em dentes contaminados por S.intermedius, entre uma coleta inicial e uma coleta realizada 7 dias após o PQC e que o aumento da dose de energia do laser aplicada e do tempo pré-irradiação não levaram a maior redução dessa contagem. Bergmans et al. (2008) encontraram redução de $93,8 \%$ em dentes contaminados com S. anginosus, de $88,4 \%$ em dentes contaminados com E. faecalis e de $98,5 \%$ nos contaminados por $F$. nucleatum, o que significou uma redução estatisticamente significativa em relação à amostra inicial. Fonseca et al. (2008) encontraram redução da contagem bacteriana de dentes contaminados por E. faecalis de 99,9\%, numa coleta realizada 7 dias após o experimento.

Nos estudos in vivo, também se avaliou a eficácia adicional da PDT. Bonsor et al. (2006) realizaram o PQC com instrumentos rotatórios de NiTi do sistema Profile e irrigação alternada de $\mathrm{NaOCl}$ a 2,25\% e ácido cítrico a 20\%. Em seguida, realizaram-se a TFD com azul de toluidina deixado no canal por um minuto, e com o laser com $100 \mathrm{~mW}$ de potência por dois minutos. Fez-se apenas uma análise quantitativa dos resultados, em que se observou que, dos quatro canais que ainda estavam contaminados, três obtiveram cultura negativa após a PDT, numa coleta realizada três dias após o PQC.

O resultado obtido neste estudo pelo grupo Tan, em que o tanino hidrolizável foi utilizado sozinho ao final do PQC, mostra que esta substância não é tão eficaz na redução microbiana, quando comparada com a sua utilização como fotossensibilizador na PDT, como coadjuvante ao uso do $\mathrm{NaOCl}$ a 2,5\% no PQC. Assim como os grupos PDT/AM e PDT/Tan, em que houve uma redução de UFC's, esta não foi tão eficaz quanto à associação da PDT com o $\mathrm{NaOCl}$ a 2,5\%, como nos grupos $\mathrm{NaOCl} / \mathrm{PDT} / \mathrm{Tan}$ e NaOCl/PDT/AM, mostrando que a PDT e o FS não são eficazes sozinhos, como no estudo de Bergman et al. (2009). A redução microbiana nos grupos PDT/AM, Tan e PDT/Tan pode ser decorrente da própria ação mecânica do instrumento endodôntico nas paredes do SCR e não do potencial antimicrobiano apresentado pelas substâncias.

No presente estudo verificou-se uma redução de 98,33\% entre as coletas inicial e final, enquanto que a redução de UFC's utilizando-se o azul de metileno como FS na PDT, foi de 88,76\%, sendo, portanto, inferior ao tanino hidrolizável, mostrando diferença estatisticamente significativa quando foi utilizado o tanino como FS na PDT coadjuvante ao PQC. Tal resultado pode estar fundamentado no fato da molécula do tanino hidrolizável apresentar união com resíduo de glicose central, 
aumentando a sua solubilidade e a ligação da molécula às lipoproteínas de baixa densidade, tornando mais fácil a entrada na célula-alvo, executando com maior eficácia a sua função de reduzir UFC's. Tais propriedades foram observadas em um estudo, onde o tanino hidrolizável, utilizado como FS na PDT mostrou-se eficaz para o tratamento de degeneração macular relacionada à idade e câncer de próstata (Verza et al., 2012).

Neste estudo, que obteve resultados diversos do estudo de Garcez et al. (2006), e semelhante ao resultado do estudo de Seal et al. (2002), quanto ao fato do $\mathrm{NaOCl}$ a 2,5\% ter se mostrado mais eficaz na redução microbiana entre as coletas inicial e intermediária, quando comparado à sua associação com a PDT, reitera-se a utilização desta solução irrigadora em tratamentos endodônticos realizados em sessão única.

Porém, em casos de retratamento endodôntico, ou de infecções persistentes, onde a quantidade de Enterococcus faecalis no interior do sistema de canais radiculares é maior e existe a necessidade de múltiplas sessões, a associação da PDT, como coadjuvante ao uso do $\mathrm{NaOCl}$ a 2,5\% no PQC torna-se mais eficaz, sobretudo utilizando-se o tanino hidrolizável como fotossensibilizador. Neste estudo, tal substância mostrou-se mais eficaz que o azul de metileno, pelo fato de ter fácil obtenção, encontram-se presentes em inúmeros vegetais, apresentam boas propriedades físico-químicas como melhor difusão entre as membranas celulares, tornando-se uma alternativa dentre os corantes utilizados na Terapia Fotodinâmica.

\section{Conclusão}

O tanino hidrolizável como fotossensibilizador na PDT mostrou-se mais eficaz que o azul de metileno na redução microbiana em canais de dentes bovinos contaminados com Enterococcus faecalis após 7 dias, como coadjuvante ao preparo químico-cirúrgico com hipoclorito de sódio a 2,5\%. Diante dos resultados promissores, há a necessidade de novos estudos, experimentais e clínicos, empregando-se outros lasers e parâmetros.

\section{Referências}

Asongalem, E. A., Foyet, H. S., Ngogang, J., Folefoc, G. N., Dimo, T., \& Kamtchouing, P. (2004). Analgesic and antiinflammatory activities of Erigeron floribundus. Journal of Ethnopharmacology, 91(2-3), 301-308. https://doi.org/10.1016/j.jep.2004.01.010

Bergmans, L., Moisiadis, P., Huybrechts, B., Van Meerbeek, B., Quirynen, M., \& Lambrechts, P. (2008). Effect of photo-activated disinfection on endodontic pathogens ex vivo [Journal Article; Randomized Controlled Trial; Research Support, Non-U.S. Gov't]. International endodontic journal, 41(3), 227-239. https://doi.org/10.1111/j.1365-2591.2007.01344.x

Bonsor, S. J., Nichol, R., Reid, T. M., \& Pearson, G. J. (2006). Microbiological evaluation of photo-activated disinfection in endodontics (an in vivo study). Brazilian Dental Journal, 200(6), 337-341, discussion 329. https://doi.org/10.1038/sj.bdj.4813371

Bonsor, S. J., \& Pearson, G. J. (2006). Current clinical applications of photo-activated disinfection in restorative dentistry. Dental Update, 33(3), 143-144, 147150, 153. https://doi.org/10.12968/denu.2006.33.3.143

Carson, K. R., Goodell, G. G., \& McClanahan, S. B. (2005). Comparison of the antimicrobial activity of six irrigants on primary endodontic pathogens. Journal of Endodontics, 31(6), 471-473. https://www.ncbi.nlm.nih.gov/pubmed/15917691

Dametto, F. R., Ferraz, C. C., Gomes, B. P., Zaia, A. A., Teixeira, F. B., \& de Souza-Filho, F. J. (2005). In vitro assessment of the immediate and prolonged antimicrobial action of chlorhexidine gel as an endodontic irrigant against Enterococcus faecalis. Oral Surgery, Oral Medicine, Oral Pathology and Oral Radiology, 99(6), 768-772. https://doi.org/10.1016/j.tripleo.2004.08.026

Evans, M., Davies, J. K., Sundqvist, G., \& Figdor, D. (2002). Mechanisms involved in the resistance of Enterococcus faecalis to calcium hydroxide. International Endodontic Journal, 35(3), 221-228. https://doi.org/10.1046/j.1365-2591.2002.00504.x

Fimple, J. L., Fontana, C. R., Foschi, F., Ruggiero, K., Song, X., Pagonis, T. C., Tanner, A. C., Kent, R., Doukas, A. G., Stashenko, P. P., \& Soukos, N. S. (2008, Jun). Photodynamic treatment of endodontic polymicrobial infection in vitro. Journal of Endodontics, 34(6), 728-734. https://doi.org/10.1016/j.joen.2008.03.011

Fonseca, M. B., Júnior, P. O., Pallota, R. C., Filho, H. F., Denardin, O. V., Rapoport, A., Dedivitis, R. A., Veronezi, J. F., Genovese, W. J., \& Ricardo, A. L. (2008). Photodynamic therapy for root canals infected with Enterococcus faecalis. Photomedicine and Laser Surgery, 26(3), 209-213. https://doi.org/10.1089/pho.2007.2124

Garcez, A. S., Nuñez, S. C., Hamblin, M. R., \& Ribeiro, M. S. (2008). Antimicrobial effects of photodynamic therapy on patients with necrotic pulps and periapical lesion. Journal of Endodontics, 34(2), 138-142. https://doi.org/10.1016/j.joen.2007.10.020 
Garcez, A. S., Nunez, S. C., Lage-Marques, J. L., Hamblin, M. R., \& Ribeiro, M. S. (2007). Photonic real-time monitoring of bacterial reduction in root canals by genetically engineered bacteria after chemomechanical endodontic therapy. Brazilian Dental Journal, 18(3), 202-207. https://doi.org/10.1590/s010364402007000300005

Gomes, B. P., Ferraz, C. C., Vianna, M. E., Berber, V. B., Teixeira, F. B., \& Souza-Filho, F. J. (2001). In vitro antimicrobial activity of several concentrations of sodium hypochlorite and chlorhexidine gluconate in the elimination of Enterococcus faecalis. Internaitonal Endodontic Journal, 34(6), 424-428. https://doi.org/10.1046/j.1365-2591.2001.00410.x

Gomes, B. P., Pinheiro, E. T., Jacinto, R. C., Zaia, A. A., Ferraz, C. C., \& Souza-Filho, F. J. (2008). Microbial analysis of canals of root-filled teeth with periapical lesions using polymerase chain reaction. Journal of Endodontics, 34(5), 537-540. https://doi.org/10.1016/j.joen.2008.01.016

Gomes, B. P., Souza, S. F., Ferraz, C. C., Teixeira, F. B., Zaia, A. A., Valdrighi, L., \& Souza-Filho, F. J. (2003). Effectiveness of 2\% chlorhexidine gel and calcium hydroxide against Enterococcus faecalis in bovine root dentine in vitro. International Endodontic Journal, 36(4), 267-275. https://doi.org/10.1046/j.1365-2591.2003.00634.x

Konopka, K., \& Goslinski, T. (2007). Photodynamic therapy in dentistry. Journal of Dental Research, 86(8), 694-707. https://doi.org/10.1177/154405910708600803

Menezes, M. M., Valera, M. C., Jorge, A. O., Koga-Ito, C. Y., Camargo, C. H., \& Mancini, M. N. (2004). In vitro evaluation of the effectiveness of irrigants and intracanal medicaments on microorganisms within root canals. International Endodontic Journal, 37(5), 311-319. https://doi.org/10.1111/j.01432885.2004.00799.x

Orstavik, D. (1996). Time-course and risk analyses of the development and healing of chronic apical periodontitis in man. International Endodontic Journal, 29(3), 150-155. https://doi.org/10.1111/j.1365-2591.1996.tb01361.x

Seal, G. J., Ng, Y. L., Spratt, D., Bhatti, M., \& Gulabivala, K. (2002). An in vitro comparison of the bactericidal efficacy of lethal photosensitization or sodium hyphochlorite irrigation on Streptococcus intermedius biofilms in root canals. International Endodontic Journal, 35(3), 268-274. https://doi.org/10.1046/j.13652591.2002.00477.x

Silva Garcez, A., Nunez, S. C., Lage-Marques, J. L., Jorge, A. O., \& Ribeiro, M. S. (2006). Efficiency of NaOCl and laser-assisted photosensitization on the reduction of Enterococcus faecalis in vitro. Oral Surgery, Oral Medicine, Oral Pathology and Oral Radiology, 102(4), e93-98. https://doi.org/10.1016/j.tripleo.2006.02.015

Siqueira, J. F., Jr., Machado, A. G., Silveira, R. M., Lopes, H. P., \& de Uzeda, M. (1997, Jul). Evaluation of the effectiveness of sodium hypochlorite used with three irrigation methods in the elimination of Enterococcus faecalis from the root canal, in vitro. International Endodontic Journal, 30(4), 279-282. https://doi.org/10.1046/j.1365-2591.1997.00096.x

Siqueira, J. F., Jr., \& Rocas, I. N. (2005). Exploiting molecular methods to explore endodontic infections: Part 2--Redefining the endodontic microbiota. Journal of Endodontics, 31(7), 488-498. https://doi.org/10.1097/01.don.0000157990.86638.49

Siren, E. K., Haapasalo, M. P., Ranta, K., Salmi, P., \& Kerosuo, E. N. (1997). Microbiological findings and clinical treatment procedures in endodontic cases selected for microbiological investigation. International Endodontic Journal, 30(2), 91-95. https://www.ncbi.nlm.nih.gov/pubmed/10332242

Soukos, N. S., Chen, P. S., Morris, J. T., Ruggiero, K., Abernethy, A. D., Som, S., Foschi, F., Doucette, S., Bammann, L. L., Fontana, C. R., Doukas, A. G., \& Stashenko, P. P. (2006). Photodynamic therapy for endodontic disinfection. Journal of Endodontics, 32(10), 979-984. https://doi.org/10.1016/j.joen.2006.04.007

Soukos, N. S., Ximenez-Fyvie, L. A., Hamblin, M. R., Socransky, S. S., \& Hasan, T. (1998). Targeted antimicrobial photochemotherapy. Antimicrobial Agents and Chemotherapy, 42(10), 2595-2601. https://doi.org/10.1128/AAC.42.10.2595

Verza, S. G., Silveira, F., Cibulski, S., Kaiser, S., Ferreira, F., Gosmann, G., Roehe, P. M., \& Ortega, G. G. (2012). Immunoadjuvant activity, toxicity assays, and determination by UPLC/Q-TOF-MS of triterpenic saponins from Chenopodium quinoa seeds. Journal of Agricultural and Food Chemistry, 60(12), 31133118. https://doi.org/10.1021/jf205010c

Wainwright, M. (2008). Photodynamic therapy: the development of new photosensitisers. Anti-Cancer Agents in Medicinal Chemistry, 8(3), 280-291. https://doi.org/10.2174/187152008783961888

Williams, J. A., Pearson, G. J., \& Colles, M. J. (2006). Antibacterial action of photoactivated disinfection \{PAD\} used on endodontic bacteria in planktonic suspension and in artificial and human root canals. Journal of Dentistry, 34(6), 363-371. https://doi.org/10.1016/j.jdent.2005.08.002

Zoletti, G. O., Siqueira, J. F., \& Santos, K. R. (2006). Identification of Enterococcus faecalis in root-filled teeth with or without periradicular lesions by culturedependent and-independent approaches. Journal of Endodontics, 32(8), 722-726. https://doi.org/10.1016/j.joen.2006.02.001 